\title{
miR-494 is an independent prognostic factor and promotes cell migration and invasion in colorectal cancer by directly targeting PTEN
}

\author{
HAI-BING SUN, XI CHEN, HONG JI, TAO WU, HONG-WEI LU, \\ YAN ZHANG, HUA LI and YI-MING LI \\ Department of General Surgery, The Second Affiliated Hospital \\ of Xi'an Jiaotong University, Xi'an, Shaanxi 710004, P.R. China
}

Received July 4, 2014; Accepted September 5, 2014

DOI: $10.3892 /$ ijo.2014.2665

\begin{abstract}
Accumulating evidence has shown that microRNAs (miRNAs) are involved in multiple processes in cancer development and progression. Upregulation of miRNA-494 (miR-494) has been identified as an oncogenic miRNA and is associated with poor prognosis in several types of human cancer. However, the specific function of miR-494 in colorectal cancer remains unclear. In this study we found that the expression of miR-494 in colorectal cancer tissues and cell lines was much higher than in normal control tissues and cells, respectively. In addition, upregulation of miR-494 more frequently occurred in tissue specimens with adverse clinical stage and the presence of distant metastasis. Moreover, multivariate survival analyses demonstrated that overexpression of miR-494 is an independent prognostic factor for both progression-free and overall survival. In addition miR-494 promoted invasion and migration in colorectal cancer cells, and miR-494 directly inhibited the phosphatase and tensin homolog deleted on chromosome 10 (PTEN) expression by targeting its 3'-untranslated region (3'-UTR). Moreover, PTEN is down regulated and inversely correlated with miR-494 expression in tissues. Thus, for the first time, we provided convincing evidence that upregulation of miR-494 was associated with tumor aggressiveness and tumor metastasis and promoted cell
\end{abstract}

Correspondence to: Professor Yi-Ming Li or Dr Hai-Bing Sun, Department of General Surgery, The Second Affiliated Hospital of Xi'an Jiaotong University, 157 Xiwu Road, Xi'an, Shaanxi 710004, P.R. China

E-mail: 1ym710004@163.com

E-mail: soloosun@163.com

Abbreviations: miR-494, miRNA-494; PTEN, phosphatase and tensin homolog deleted on chromosome 10; WT, wild-type; MT, mutant; 3'-UTR, 3'-untranslated region; RT-qPCR, quantitative real-time polymerase chain reaction

Key words: miR-494, colorectal cancer, metastasis, prognosis, PTEN migration and invasion by targeting PTEN gene in colorectal cancer, and miR-494 is an independent prognostic marker for colorectal cancer patients.

\section{Introduction}

Colorectal carcinoma (CRC) is the third most frequently diagnosed malignancy and the third leading cause of death among cancer patients in the United States (1). Despite current therapeutic strategies combining adjuvant chemotherapy, surgery and sometimes radiotherapy, the prognosis of CRC remains poor, since most CRC patients have distant metastases at diagnosis or develop recurrent metastatic CRC following surgical treatment. Although recent developments in molecular biology have provided insight into the molecular mechanisms of CRC, the fundamental molecular mechanisms underlying metastasis in CRC have not been fully elucidated. Therefore, it is essential to identify metastasis-associated molecules as effective drug targets and to enhance the understanding of the mechanisms underlying the metastasis of CRC.

MicroRNAs (miRNAs) are small non-coding RNAs ( $\sim 22$ nucleotides in length), transcribed from non-protein-coding genes or introns, which regulate gene expression through repressing translation and cleaving their mRNAs by binding to complementary sites in their 3'-untranslated region (3'-UTR) (2). miRNAs regulated the expression of a wide variety of target genes, and aberrant expression miRNAs cause them to function as tumor suppressors or oncogenes according to the role of their target genes $(3,4)$. Particularly, miRNAs can regulate various biological processes of tumor cells, including cell proliferation, differentiation, progression, apoptosis, proliferation, migration and invasion (5-7). Furthermore, increasing numbers of miRNAs have been observed in various types of cancer and may be involved in modulating cancer cell behavior (8-10). Several aberrantly expressed miRNAs have been proven to be associated with tumorigenesis, tumor progression and metastasis in $\mathrm{CRC}$, and taken as prognostic indicators for CRC including miR-150 (11), miR-28-5p/-3p (12), miR-200 (13) and many others (14-16).

miRNA-494 (miR-494), located on chromosome 14q32.31, has been reported to be highly expressed in retinoblastoma 
cells (17) and in the anti-BPDE-induced human bronchial epithelial cell line $16 \mathrm{HBE}(18)$, but the role of miR-494 in carcinogenesis is still not fully understood. In tumor-expanded myeloid-derived suppressor cells, it has been reported that miR-494 was able to regulate the expression phosphatase and tensin homolog deleted on chromosome 10 (PTEN) post-transcriptionally and function as a micro-oncogene in carcinogenesis (19). In glioma, miR-494 was also proved to be a target to control invasiveness in cancer therapy (20). These studies suggest that miR-494 may play an important oncogenic role in tumorigenesis and metastasis. But miR-494 was found downregulated and suppressed cell proliferation in gastric cancer, A549 lung cancer cells cholangiocarcinoma (21-23). It is still unclear whether miR-494 was an oncogenic miRNA or a tumor suppressor miRNA in CRC. Thus the role of miR-494 in carcinogenesis and metastasis of CRC needed further investigations.

The aim of our study was to investigate the effects of miR-494 on cell migration and invasion and further discuss the mechanism of action of miR-494 by identifying its potential target gene in CRC. We found that miR-494 expression was upregulated in the majority of CRC tissues, and upregulated miR-494 was significantly associated with tumor recurrence, metastasis and poor prognosis in CRC patients. Moreover, in vitro assays showed that miR-494 could promote tumor migration and invasion of CRC cell lines by targeting its direct target PTEN. To the best of our knowledge, this is the first study to examine the expression and role of miR-494 in prognosis and metastasis of CRC.

\section{Materials and methods}

Patients and tissue samples. This study was approved by the Research Ethics Committee of Xi'an Jiaotong University. Written informed consent was obtained from all the patients. The specimens were handled and made anonymous according to the ethical and legal standards. A total of 247 patients were enrolled in this study. Patients received curative resection for CRC at the Second Affiliated Hospital, Xi'an JiaoTong University (Shaanxi, China) between 2001-2008. None of the patients enrolled in this research received blood transfusion or chemotherapy before surgery. The clinicopathological information of the patients is shown in Table I. The follow-up information of all participants was updated every 3 months by telephone. The overall survival was defined as the time elapsed from surgery to death. Information regarding the death of patients was ascertained from their family. Patients were followed after surgical treatment until May 2013, with a median follow-up of 83 months (range, 8-139 months). During the follow-up period, 121 patients $(49.0 \%)$ died of the disease. The median overall and progression-free survival of patients was 37 and 34 months, respectively.

Quantitative reverse transcriptase $P C R(R T-q P C R)$ assay. The expression of miR-494 in CRC and corresponding adjacent tissues were detected by RT-qPCR assay. Briefly, total RNA was extracted from tissues using TRIzol reagent (Invitrogen Life Technologies, Carlsbad, CA, USA) according to the manufacturer's protocol. Then, miRNA expression levels were quantitated using TaqMan microRNA real-time RT-PCR
Table I. Clinical correlation between miR-494 expression and other clinicopathological features in CRC.

\begin{tabular}{|c|c|c|c|c|}
\hline $\begin{array}{l}\text { Clinicopathological } \\
\text { features }\end{array}$ & $\begin{array}{l}\text { Total no. } \\
\text { of patients }\end{array}$ & $\begin{array}{l}\text { High miR-494 } \\
\text { group }\end{array}$ & $\chi^{2}$ & $\mathrm{P}$ \\
\hline Age (years) & $247(57.4 \pm 10.7)$ & 129 & 0.612 & 0.287 \\
\hline$\leq 60$ & 117 & 59 & & \\
\hline$>60$ & 130 & 70 & & \\
\hline Sex & & & 0.002 & 0.962 \\
\hline Men & 149 & 78 & & \\
\hline Women & 98 & 51 & & \\
\hline Tumor size & & & 2.468 & 0.116 \\
\hline$\leq 5 \mathrm{~cm}$ & 171 & 95 & & \\
\hline$>5 \mathrm{~cm}$ & 76 & 34 & & \\
\hline Location & & & 0.045 & 0.832 \\
\hline Colon & 167 & 88 & & \\
\hline Rectum & 80 & 41 & & \\
\hline Depth of invasion & & & 0.118 & 0.732 \\
\hline $\mathrm{T} 1-\mathrm{T} 2$ & 79 & 40 & & \\
\hline $\mathrm{T} 3-\mathrm{T} 4$ & 168 & 89 & & \\
\hline Lymph node status & & & 7.705 & $0.006^{\mathrm{a}}$ \\
\hline Negative & 145 & 65 & & \\
\hline Positive & 102 & 64 & & \\
\hline Metastasis status & & & 10.554 & $0.001^{\mathrm{a}}$ \\
\hline Negative & 203 & 101 & & \\
\hline Positive & 44 & 28 & & \\
\hline TNM stage & & & 9.163 & $0.027^{\mathrm{a}}$ \\
\hline I & 42 & 15 & & \\
\hline II & 73 & 39 & & \\
\hline III & 88 & 45 & & \\
\hline IV & 44 & 30 & & \\
\hline Differentiation & & & 7.650 & $0.022^{\mathrm{a}}$ \\
\hline Well & 119 & 53 & & \\
\hline Moderately & 76 & 41 & & \\
\hline Poorly & 52 & 35 & & \\
\hline
\end{tabular}

${ }^{\mathrm{a}} \mathrm{P} \leq 0.05$. miR-494, miRNA-494; CRC, colorectal carcinoma.

kit (Applied Biosystems, Foster City, CA, USA) according to the manufacturer's protocol. Data were analyzed with 7500 Software v.2.0.1 (Applied Biosystems), with the automatic $\mathrm{Ct}$ setting for adapting baseline and threshold for Ct determination. The universal small nuclear RNA U6 (RNU6B) was used as an endogenous control for miRNAs. Each sample was examined in triplicate and the amounts of PCR products produced were non-neoplasticized to RNU6B.

Cell culture. Human CRC cell lines SW620,SW480,HCT116 and normal colon epithelium cell line CCD-18Co were obtained from the Cell Bank of the Chinese Academy of Sciences (Shanghai, China), where they were characterized by mycoplasma detection, DNA fingerprinting, isozyme detection and cell vitality detection. They were cultured in RPMI-1640 (Invitrogen Life 

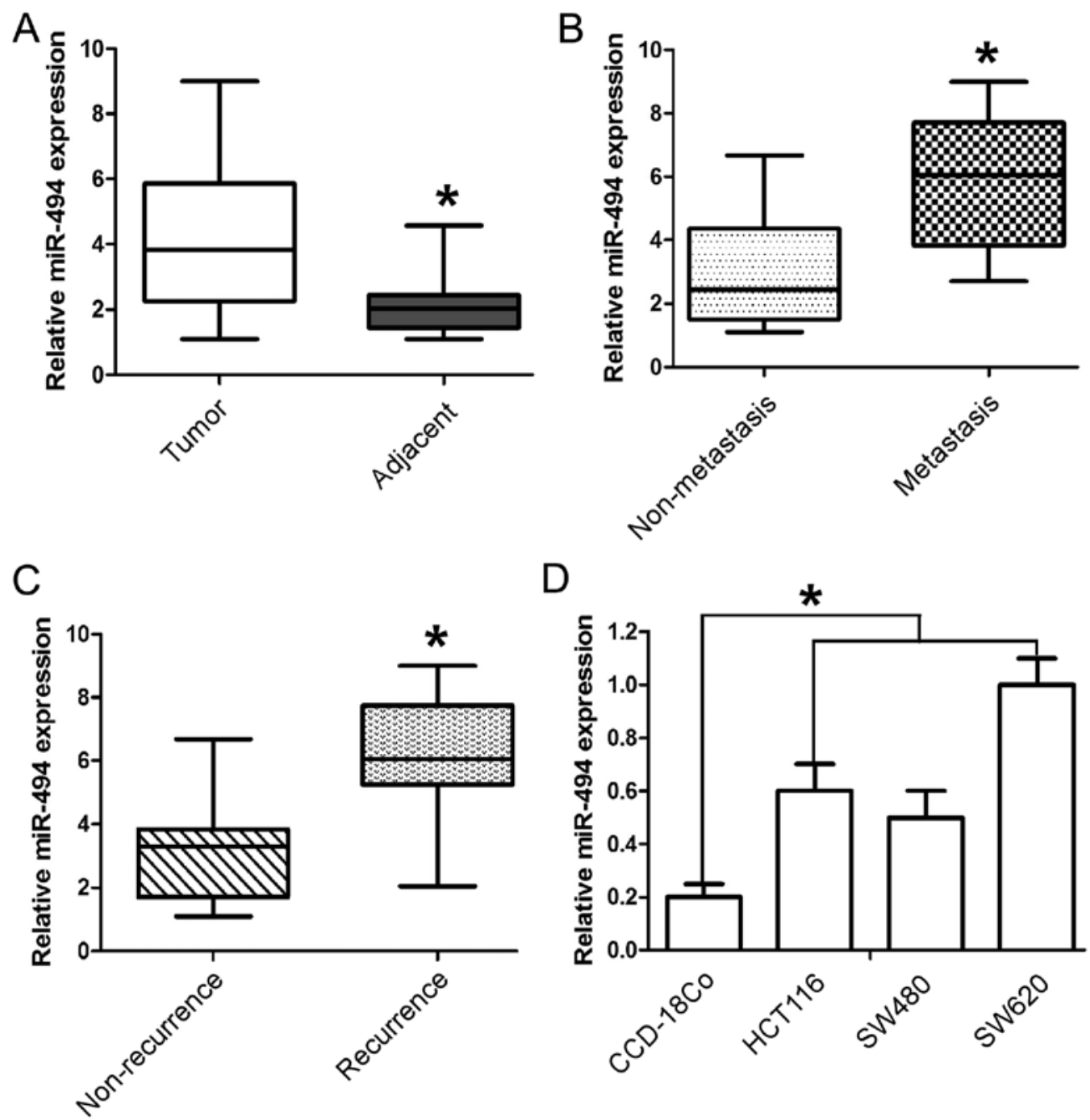

Figure 1. The expression levels of miRNA-494 (miR-494) in colorectal carcinoma (CRC) tissues and cell lines. (A) The relative expression of miR-494 in 247 paired human CRC and their corresponding adjacent samples. (B) Comparison of the expression of miR-494 between metastatic and non-metastatic CRC tissues. (C) Comparison of the expression of miR-494 between tumor tissues from recurrent and non-recurrent group. (D) The relative expression levels of miR-494 in CRC cell lines and human normal colon epithelium cell line CCD-18Co. Expression levels of miR-494 were determined by RT-qPCR and normalized against an endogenous control U6 RNA. " $\mathrm{P}<0.05$.

Technologies) mediums supplemented with $10 \%$ fetal bovine serum (FBS) (HyClone, Logan, UT, USA) and cultured in a humidified incubator at $37^{\circ} \mathrm{C}$ in $5 \% \mathrm{CO}_{2}$.

Oligonucleotide transfection. miR-494 mimics and inhibitors were chemically synthesized by Shanghai GenePharma (Shanghai, China). Once the cells were $80 \%$ confluent, miR-494 mimics or inhibitor was transfected into osteosarcoma cells with Lipofectamine 2000 (Invitrogen Life Technologies) according to the manufacturer's instructions. Cells were also transfected with scramble oligonucleotide as negative control (NC). The expression level of miR-494 in the transfected osteosarcoma cells were identified by RT-qPCR.

In vitro migration and invasion assays. Cell migration and invasion capacity were measured using Transwell migration assays (Millipore, Billerica, MA, USA) in vitro. The CRC SW620 cells were transfected with miR-494 inhibitors and NCs for $48 \mathrm{~h}$, the CRC SW480 cells were transfected with miR-494 mimics and NCs for $48 \mathrm{~h}$, and then the cells were suspended in RPMI-1640 with $10 \mathrm{~g} / 1 \mathrm{BSA}$ at a density of
$1 \times 10^{6}$ cells $/ \mathrm{ml}$. Then, cell suspensions $(150 \mu \mathrm{l})$ were seeded in the upper chamber with aporous membrane coated with (for the Transwell invasion assay) or without (for the migration assay) Matrigel (BD Biosciences, San Diego, CA, USA). To attract the cells, $500 \mu 1$ of RPMI-1640 with $10 \%$ serum was added to the bottom chamber. After allowing the cells to migrate for $24 \mathrm{~h}$ or to invade for $48 \mathrm{~h}$, the penetrated cells on the filters were fixed in dried methanol and stained in $4 \mathrm{~g} / \mathrm{l}$ crystal violet. The numbers of migrated or invasive cells were determined from five random fields using a microscope (Olympus, Tokyo, Japan) at x10 magnification.

Luciferase reporter assay. CRC cells were seeded in a 96-well plate at $60 \%$ confluence. After $24 \mathrm{~h}$, cells were transfected with $120 \mathrm{ng}$ of wild-type (WT) or mutant (MT) 3'-UTR of PTEN mRNA expression vector pGL3-target-3'UTR and $30 \mathrm{ng}$ of miR-494 mimics using Lipofectamine 2000. Cells were collected $48 \mathrm{~h}$ after transfection, and luciferase activity was measured using a dual luciferase reporter assay system according to the manufacturer's protocol (Promega Corporation, Madison, WI, USA). 
Western blot analysis. Cells were harvested in lysis buffer (50 mM NaCl, 50 mM EDTA, 1\% Triton X-100) containing protease inhibitor cocktail (Roche Diagnostics, Indianapolis, IN, USA). The cell lysates (30 $\mu \mathrm{g})$ were separated using $10 \%$ SDS-PAGE gels and then transferred onto nitrocellulose membranes (Millipore, Bedford, MA, USA). The membranes were blocked with 5\% non-fat milk diluted in PBS for $2 \mathrm{~h}$ at room temperature before the addition of the appropriate primary antibody. The antibodies used in this study included anti-PTEN (1:1,000, no. 9188; Cell Signaling Technology, Inc., Danvers, MA, USA) and anti- $\beta$-actin (1:3,000, A2066; Sigma, St. Louis, MO, USA). The membranes were then washed with PBS containing $0.05 \%$ Tween-20 and incubated with the appropriate HRP-conjugated secondary antibody $(1: 5,000$; Sigma) for $1 \mathrm{~h}$ at room temperature. The bands were visualized using a chemiluminescence reagent (New England Nuclear, Boston, MA, USA).

Statistical analysis. Statistical analysis was performed using IBM SPSS statistical software (version 20.0). Survival curves were estimated using the Kaplan-Meier method, and distributions were evaluated by the log-rank test. Cox proportional hazard models of factors related to survival were used to calculate RRs and identify the factors that affect survival. The differences in characteristics between the two groups were examined by the $\chi^{2}$ test and Fisher's exact test. All P-values were determined from two-sided tests, and statistical significance was based on a P-value of 0.05 .

\section{Results}

Upregulation of miR-494 is associated with metastasis and recurrence of $C R C$. Expression of miR-494 was analyzed in 247 pairs of CRC and adjacent tissues by RT-qPCR and normalized against an endogenous control (U6 RNA). As shown in Fig. 1A the expression level of miR-494 in the CRC tissues was found to be distinctly upregulated when compared to that in adjacent tissues. Furthermore, in comparison to the non-metastatic CRC tissues, the miR-494 levels were significantly higher in metastatic CRC tissues (Fig. 1B). Moreover, miR-494 levels were upregulated in the tumor tissues obtained from the patients who suffered CRC recurrence (Fig. 1C). Collectively, these data indicated that significant upregulation of miR-494 expression occurred in CRC and was correlated with CRC relapse and metastasis. To further evaluate the association of miR-494 with CRC metastasis, we analyzed miR-494 levels in three CRC cell lines (SW620, SW480, and HCT116) and normal colon epithelium cell line CCD-18Co. Similarly, we found the expression of miR-494 was much higher in all three CRC cell lines than that in the normal colon cell line. It is noteworthy that miR-494 level was higher in metastatic CRC cells (SW620) than its paired metastatic cell line (SW480) (Fig. 1D). Taken together, the above findings suggest that miR-494 levels correlate with metastatic potential of CRC.

Upregulation of miR-494 is associated with advanced clinicopathological features of CRC. To determine the clinical significance of miR-494 in CRC, we analyzed the association of miR-494 expression with various clinicopathological
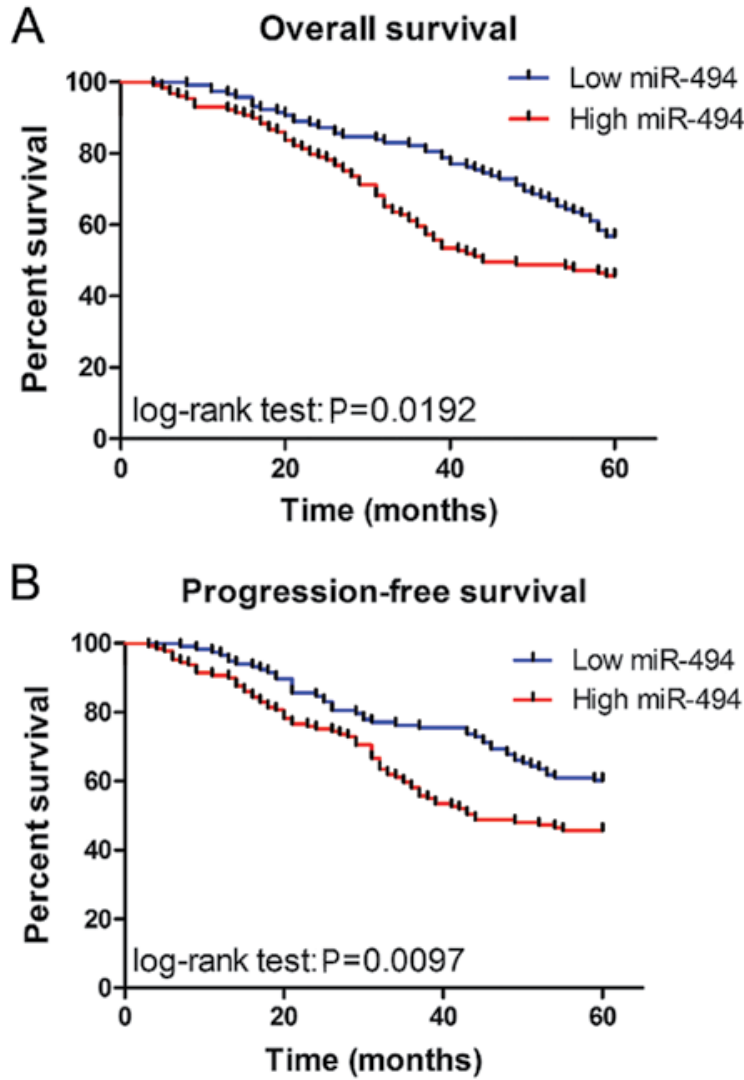

Figure 2. The relationship between miRNA-494 (miR-494) expression and overall survival or progression-free survival time. (A) Overall and (B) progression-free survival curves for two groups of low and high expression of miR-494 in patients with colorectal carcinoma (CRC).

parameters of CRC tissues. The median miR-494 expression in all 247 patients with CRC was 3.84. The patients were divided into two groups according to their expression levels of miR-494, using the median level as a cutoff: the high miR-494 expression group $(n=129)$ and the low miR-494 expression group $(n=118)$. As shown in Table I, miR-494 was significantly upregulated in CRC patients with advanced clinical stage $(\mathrm{P}=0.027)$, metastasis status $(\mathrm{P}=0.001)$, and positive lymph node metastasis $(\mathrm{P}=0.006)$. We also found a trend of increased miR-494 level from well to poor differentiation $(\mathrm{P}=0.022)$. However, miR-494 expression was not significantly correlated with gender, age, tumor size, tumor location and depth of invasion.

Upregulation of miR-494 is associated with poor prognosis in patients with CRC. To determine the prognostic value of miR-494 expression in CRC, we analyzed the relationship between the miR-494 and clinical outcome. The relationship between miR-494 expression overall survival or progression-free survival was investigated using Kaplan-Meier analysis and log-rank test. Statistically significant differences in overall survival and progression-free survival were found between the high miR-494 level group and low miR-494 level group (Fig. 2A and B; log-rank test: $\mathrm{P}=0.0192$ and $\mathrm{P}=0.0097$, respectively). The patients with high miR-494 expression tended to have shorter overall and progression-free survival time when compared to patients with low miR-494 expression. Univariate analysis identified advanced clinical stage, metastasis status, lymph node status, differentiation status and high expression of 
Table II. Cox regression analysis of prognostic factors for overall survival in colorectal cancer patients $(n=143)$.

\begin{tabular}{|c|c|c|c|c|c|c|}
\hline & \multicolumn{3}{|c|}{ Univariate } & \multicolumn{3}{|c|}{ Multivariate } \\
\hline & HR & $95 \% \mathrm{CI}$ & $\mathrm{P}$ & HR & $95 \% \mathrm{CI}$ & $\mathrm{P}$ \\
\hline miR-494 expression & 3.617 & $(1.208-4.576)$ & $0.008^{\mathrm{a}}$ & 2.874 & $(1.148-3.294)$ & $0.017^{\mathrm{a}}$ \\
\hline Age & 1.215 & $(0.478-1.935)$ & 0.617 & 1.483 & $(0.720-1.816)$ & 0.764 \\
\hline Sex & 1.415 & $(0.787-1.913)$ & 0.601 & 1.213 & $(0.547-1.811)$ & 0.538 \\
\hline Tumor location & 1.294 & $(0.567-2.104)$ & 0.711 & 0.984 & $(0.481-2.506)$ & 0.762 \\
\hline TNM stage & 2.441 & $(1.253-2.872)$ & $0.021^{\mathrm{a}}$ & 1.716 & $(1.319-2.522)$ & $0.013^{\mathrm{a}}$ \\
\hline Differentiation & 2.952 & $(1.224-2.228)$ & $0.041^{\mathrm{a}}$ & 3.291 & $(0.826-3.773)$ & 0.077 \\
\hline Tumor size & 1.761 & $(0.769-1.927)$ & 0.319 & 1.629 & $(0.813-2.109)$ & 0.427 \\
\hline Lymph node metastasis & 2.165 & $(1.481-3.251)$ & $0.014^{\mathrm{a}}$ & 1.957 & $(0.957-3.182)$ & 0.218 \\
\hline Depth of invasion & 1.291 & $(0.521-2.114)$ & 0.542 & 1.417 & $(0.619-2.217)$ & 0.611 \\
\hline Metastasis status & 2.761 & $(1.274-3.189)$ & $0.032^{\mathrm{a}}$ & 2.465 & $(1.198-2.981)$ & $0.021^{\mathrm{a}}$ \\
\hline
\end{tabular}

${ }^{\mathrm{a} P} \leq 0.05 .95 \% \mathrm{CI}, 95 \%$ confidence interval; miR-494, miRNA-494.

Table III. Cox regression analysis of prognostic factors for progression-free survival in colorectal cancer patients ( $\mathrm{n}=143$ ).

\begin{tabular}{|c|c|c|c|c|c|c|}
\hline & \multicolumn{3}{|c|}{ Univariate } & \multicolumn{3}{|c|}{ Multivariate } \\
\hline & HR & $95 \% \mathrm{CI}$ & $\mathrm{P}$ & HR & $95 \% \mathrm{CI}$ & $\mathrm{P}$ \\
\hline miR-494 expression & 4.112 & $(1.298-5.428)$ & $0.014^{\mathrm{a}}$ & 3.712 & $(1.513-5.272)$ & $0.009^{\mathrm{a}}$ \\
\hline Age & 1.461 & $(0.563-1.892)$ & 0.702 & 1.287 & $(0.641-1.674)$ & 0.815 \\
\hline Sex & 1.599 & $(0.659-2.114)$ & 0.705 & 1.761 & $(0.847-2.832)$ & 0.642 \\
\hline Tumor location & 1.379 & $(0.661-1.907)$ & 0.743 & 1.211 & $(0.575-1.803)$ & 0.619 \\
\hline TNM stage & 3.115 & $(1.418-4.861)$ & $0.034^{\mathrm{a}}$ & 2.724 & $(1.275-3.554)$ & $0.019^{\mathrm{a}}$ \\
\hline Differentiation & 2.792 & $(1.125-3.219)$ & $0.029^{\mathrm{a}}$ & 2.491 & $(0.751-3.773)$ & 0.126 \\
\hline Tumor size & 1.551 & $(0.659-1.832)$ & 0.584 & 1.441 & $(0.707-1.614)$ & 0.535 \\
\hline Lymph node metastasis & 3.144 & $(1.524-4.268)$ & $0.027^{\mathrm{a}}$ & 2.163 & $(0.897-3.264)$ & 0.144 \\
\hline Depth of invasion & 1.421 & $(0.762-2.392)$ & 0.641 & 1.721 & $(0.765-2.319)$ & 0.597 \\
\hline Metastasis status & 2.415 & $(1.103-2.891)$ & $0.013^{\mathrm{a}}$ & 2.117 & $(1.298-2.771)$ & $0.011^{\mathrm{a}}$ \\
\hline
\end{tabular}

${ }^{\mathrm{a}} \mathrm{P} \leq 0.05 .95 \% \mathrm{CI}, 95 \%$ confidence interval; miR-494, miRNA-494.

miR-494 as indicators for poor prognosis for overall survival and progression-free survival (all $\mathrm{P}<0.05$ ), whereas age, gender and tumor location were not significantly associated with overall survival and progression-free survival (Tables II and III). To test whether the prognostic value of high miR-494 expression was independent of other risk factors for poor overall and progression-free survival, a multivariate analysis was performed using a Cox proportional hazard model. Multivariate analyses including age, gender, tumor location, tumor stage, miR-494 expression, differentiation status, lymph node status, depth of invasion and metastasis status demonstrated that high miR-494 expression was an independent predictor for poor overall and progression-free survival in CRC patients $(\mathrm{HR}=2.874$, $\mathrm{CI}=1.148-3.294, \mathrm{P}=0.017$ and $\mathrm{HR}=3.712, \mathrm{CI}=1.513-5.272$, $\mathrm{P}=0.009$, respectively). Statistically significant results were also obtained for advanced tumor stage, and metastasis status, whereas all other parameters were not significant for overall and progression-free survival (Tables II and III).
miR-494 promotes the migration and invasion of CRC cells. As patients with recurrent metastatic CRC present with poor outcome, and our previous results indicated that miR-494 was significantly associated with progression-free and overall survival, we aimed to ascertain whether miR-494 affects the cell migration and invasion of CRC cells. The SW480 cell line was derived from the primary adenocarcinoma and SW620 cell line was derived from metastatic adenocarcinoma of the same patients (24). Our previous results proved that SW480 had lower expression of miR-494 than SW620. So they were used to investigate the role of miR-494 on cell migration and invasion. SW480 was transiently transfected with miR-494 mimics, whereas SW620 was transiently transfected with miR-494 inhibitors. As expected, transfection of miR-494 mimics or miR-494 inhibitors resulted in an increased or decreased miR-494 expression in SW480 or SW620, respectively (Fig. 3A and $\mathrm{B}$ ). Moreover, the migration and invasion assays demonstrated that miR-494 overexpression 


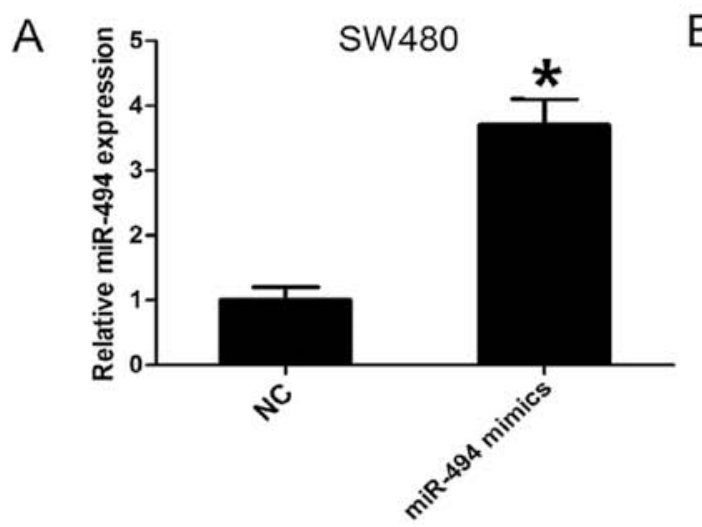

B
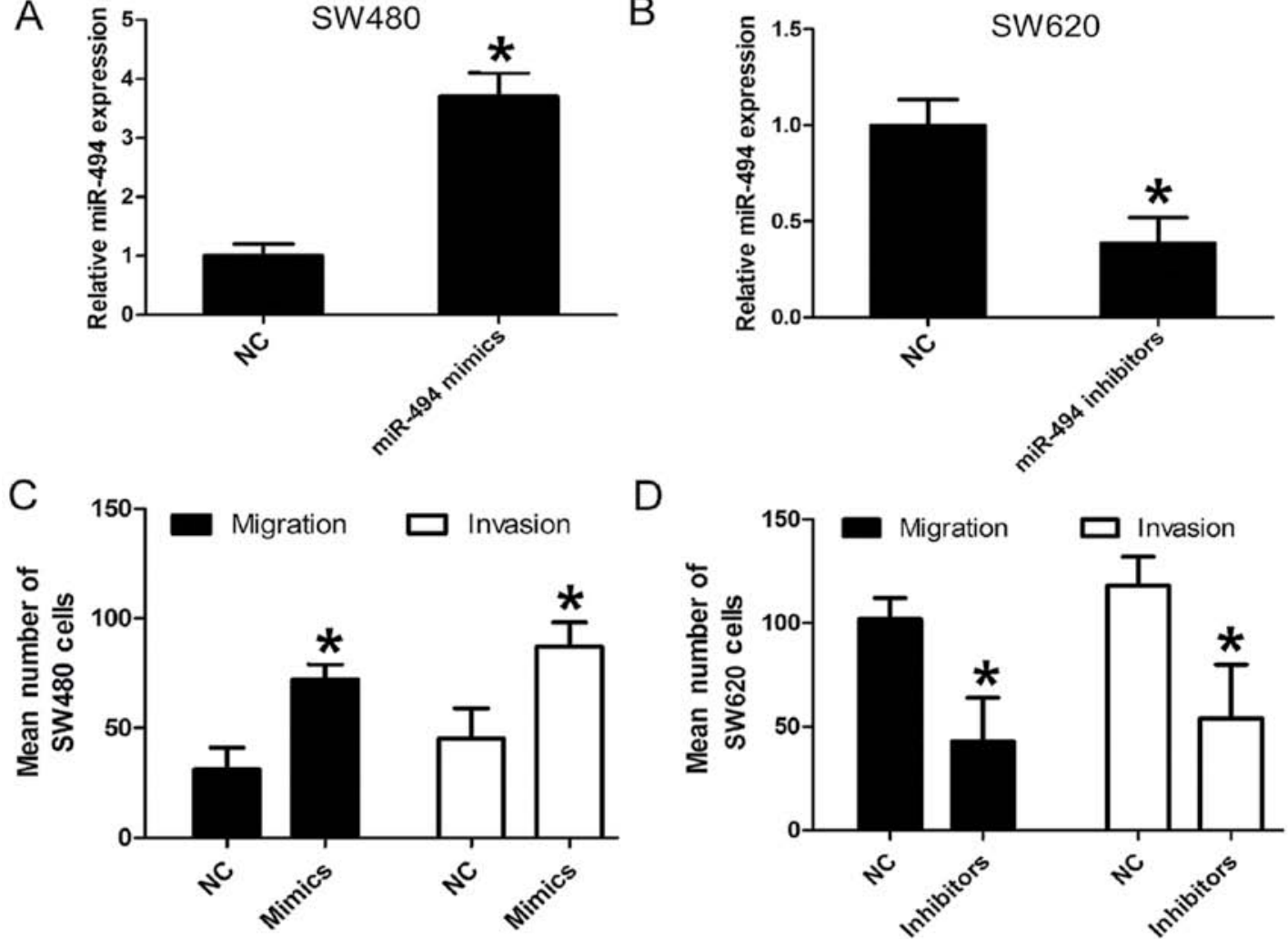

$\mathrm{D}$

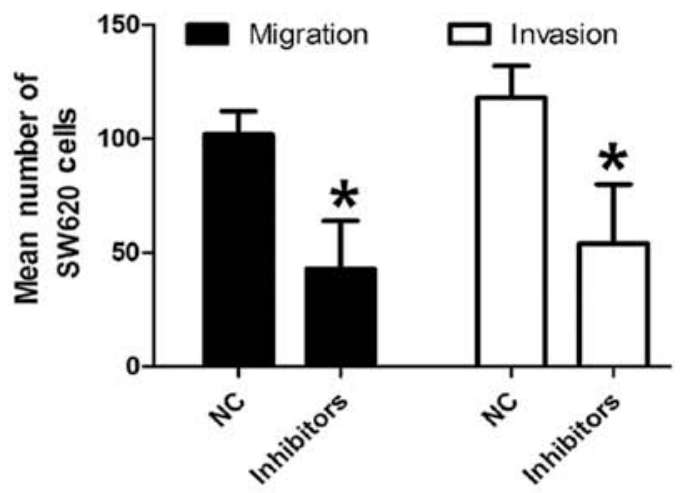

E

SW480

$\mathrm{F}$
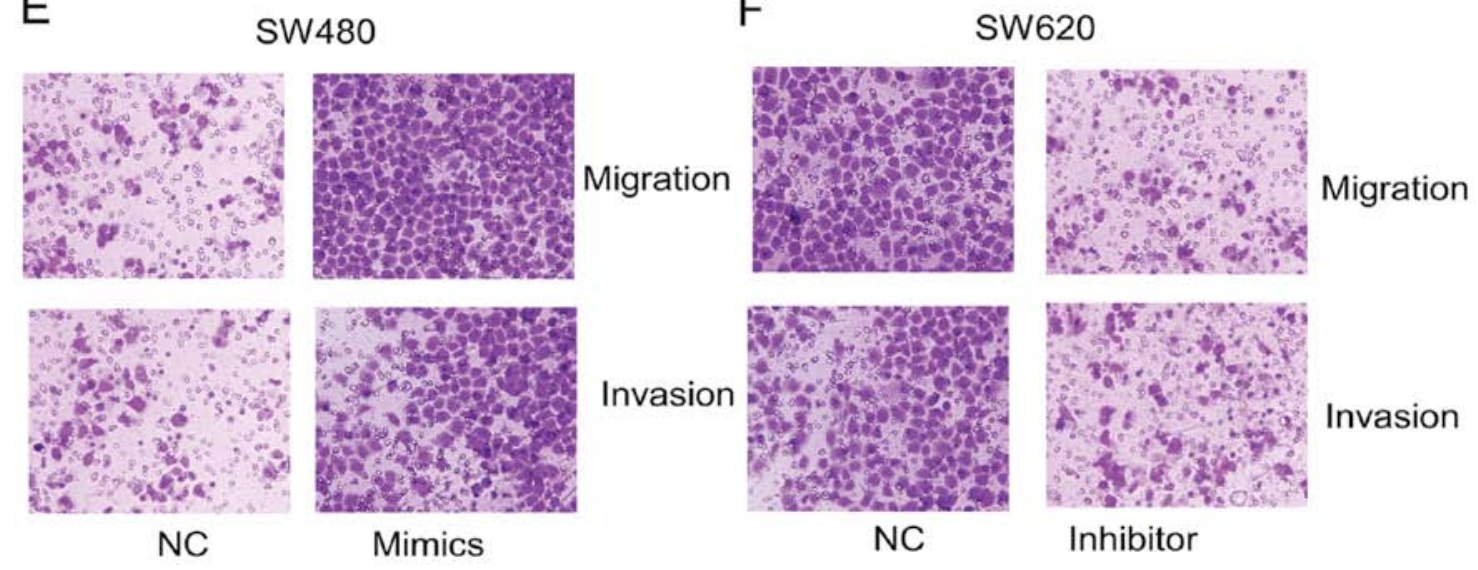

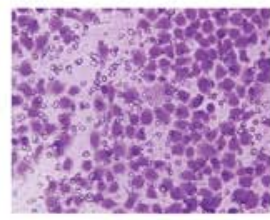

NC

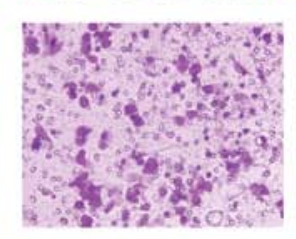

Inhibitor

Figure 3. The relationship between miRNA-494 (miR-494) expression and migration and invasion of colorectal carcinoma (CRC) cell lines. (A and B) Transfection of miR-494 mimics or inhibitors to SW480 or SW620 increases or decreases the expression of miR-494 detected by RT-qPCR, respectively. (C-F) The effect of miR-494 toward the migration and invasion of SW480 and SW620 cells. Three independent experiments were performed in duplicate. Data are presented as mean \pm SD. Two-tailed Student's t-test was used to analyze the significant differences. * $\mathrm{P}<0.05$.

significantly increased the migration and invasion ability of SW480 (Fig. 3C and E). Conversely, knockdown of miR-494 in SW620 decreased the migration and invasion ability of SW620 (Fig. 3D and F). These results indicate that miR-494 functions as oncogenic miRNA and contributes to the progression and metastasis of CRC cells.

PTEN is a direct target of miR-494 in CRC cells and inversely correlates with miR-494 in CRC tissues. To characterize the mechanism by which miR-494 promotes CRC metastasis, we searched for potential target genes of miR-494 using three publicly available databases, TargetScan, PicTar and miRanda. We were particularly interested in tumor suppressor gene $P T E N$, because PTEN was a crucial factor in various central processes of cancer development and loss of PTEN was proved to be associated with advanced CRC, liver metastasis, and poor survival $(25,26)$. It has been proven that PTEN was the direct target of miR-494 in other cells $(19,27)$. Considering the tissue-specific and developmental stage-specific manner of miRNAs, we investigate the relationship of PTEN and miR-494 in CRC cell lines. In order to confirm PTEN is a target gene for miR-494 in CRC cells, RT-qPCR was used to detect the expression of PTEN in CRC cell line SW480 and SW620. The expression of PTEN at mRNA and protein level was significantly downregulated after overexpression of miR-494 in SW480 cell line. Conversely, the expression of PTEN was significantly upregulated after knockdown miR-494 expression in SW620 cell line (Fig. 4A and B). Furthermore, we 


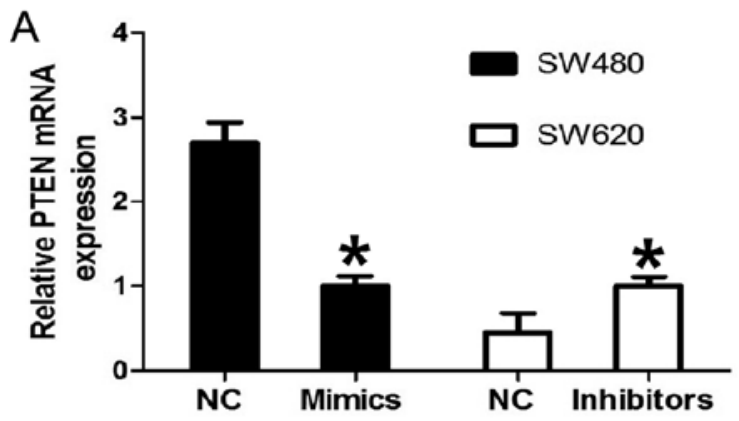

B
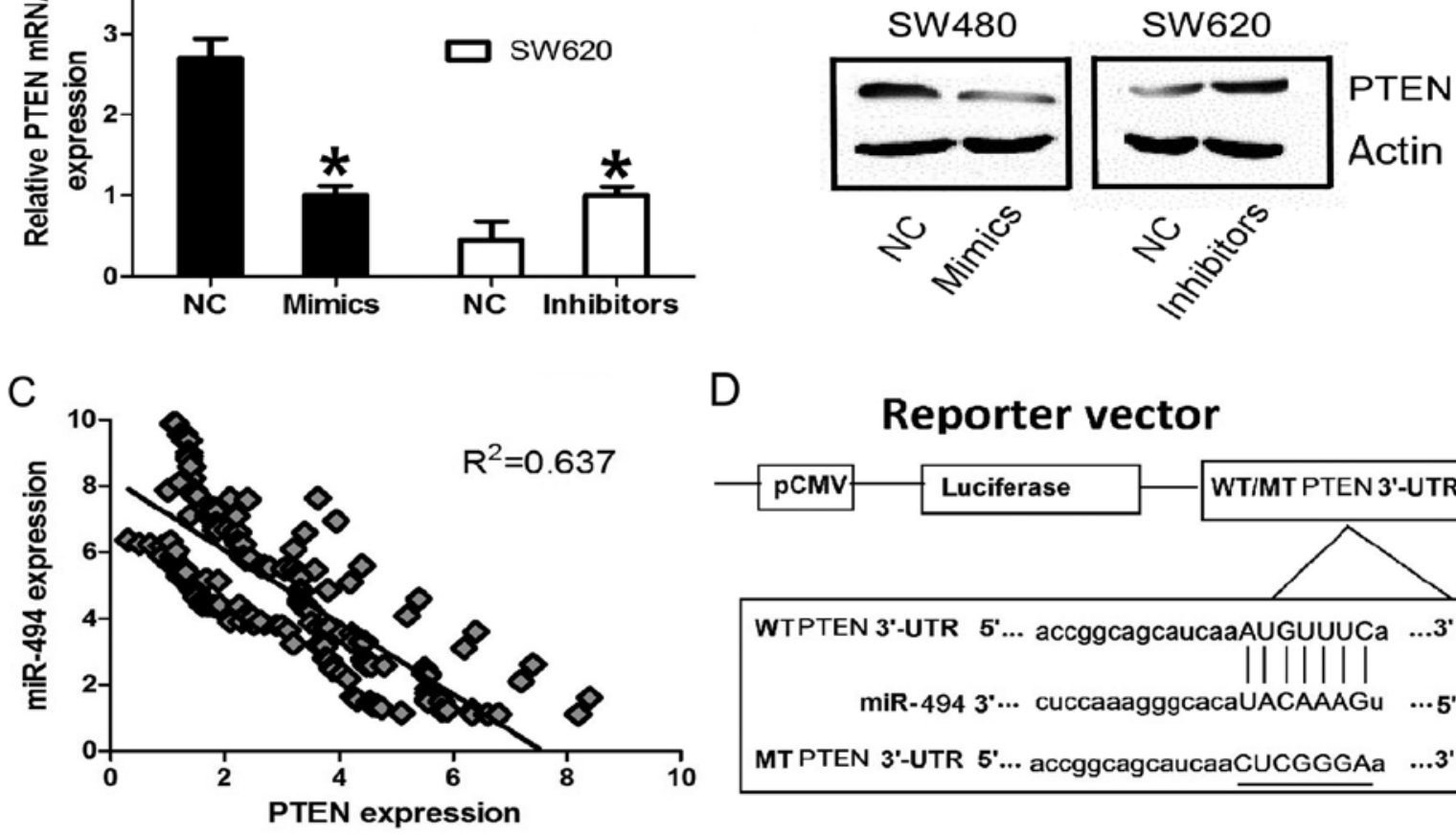

D

\section{Reporter vector}
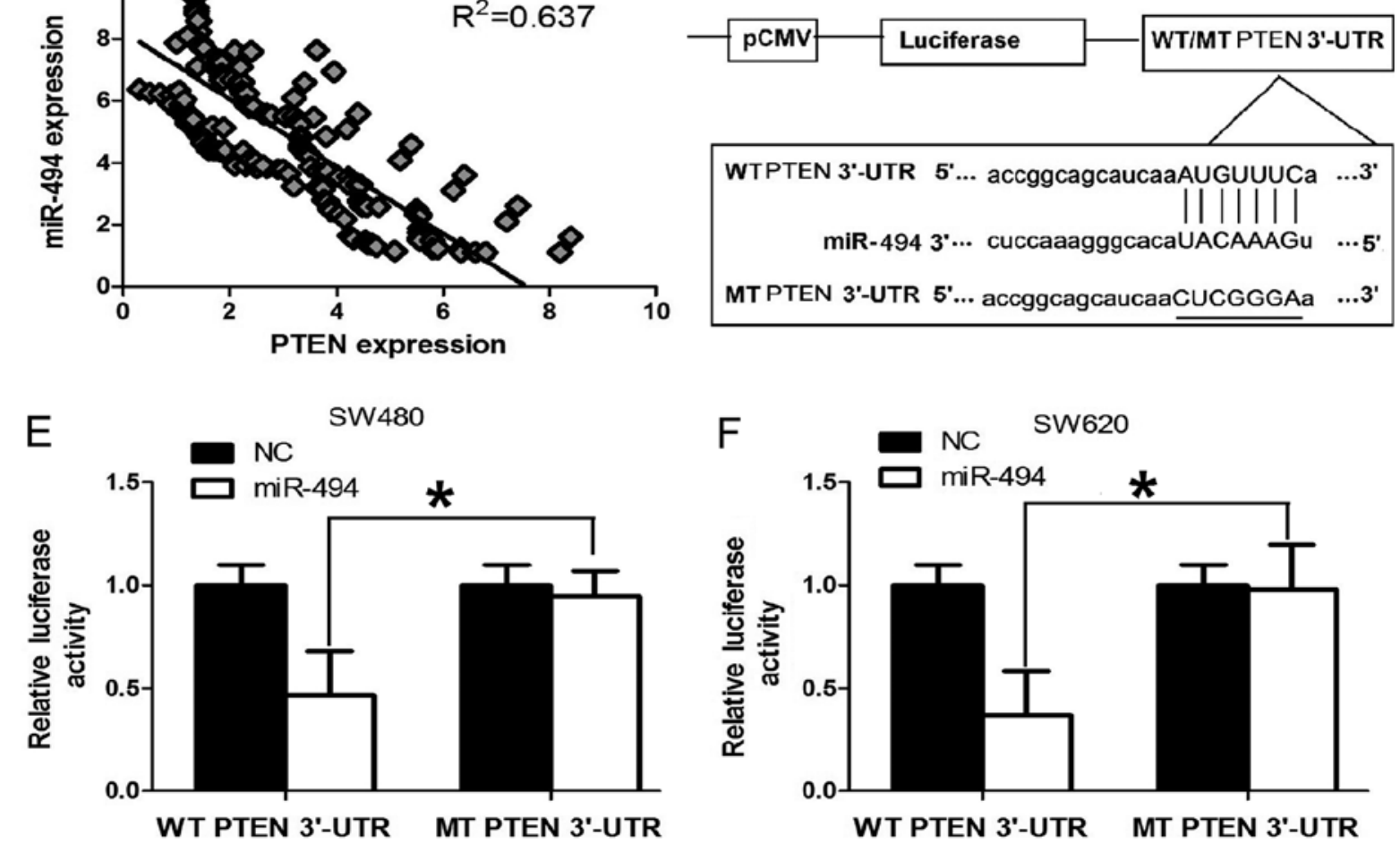

Figure 4. Phosphatase and the tensin homolog deleted on chromosome 10 (PTEN) 3'-untranslated region (3'-UTR) is the direct target of miRNA-494 (miR-494). (A and B) miR-494 mimics/inhibitors inhibited/promoted the expression level of PTEN at the mRNA level and the protein level in SW480/SW620 cells. (C) The expression of miR-494 negatively correlated with the expression of PTEN in colorectal carcinoma (CRC) tissues. (D) miR-494 and its putative binding sequence in the 3'-UTR of PTEN, and the diagram of the luciferase reporter plasmids with wild-type (WT) and mutant (MT) PTEN 3'-UTR. (E and F) The relative luciferase activity in SW480 and SW620 cells after the plasmid with WT or MT PTEN 3'-UTR co-transfected with miR-494 mimics. Three independent experiments were performed in duplicate. Data are presented as mean \pm SD. Two-tailed Student's $t$-test was used to analyze the significant differences. * $P<0.05$.

assessed the significance of the miR-494 and PTEN correlation in CRC tissues. We determined the PTEN mRNA and miR-494 expression in the same CRC specimens by RT-qPCR. As shown in Fig. 4C, a statistically significant inverse correlation was revealed by Spearman's correlation analysis between mRNA levels of miR-494 and PTEN ( $r=-0.798 ; \mathrm{P}<0.001)$. Taken together, our results suggest that miR-494 negatively regulates the expression of its potential target gene PTEN in CRC cell lines and tissues.

We further performed luciferase reporter assay to verify whether miR-101 directly targeted the 3'-UTR of PTEN in CRC cells. The target sequence of EZH2 3'-UTR (WT 3'-UTR) or the MT sequence (MT 3'-UTR) was cloned into a luciferase reporter vector (Fig. 4D). SW480 and SW620 cells were then transfected with WT or MT 3'-UTR vector and the miR-494 mimic. As shown in Fig. 4E, a significant decrease in luciferase activity was noted between the PTEN WT 3'-UTR group and the NC group in SW480 cells $(\mathrm{P}<0.05)$. The repressive effect was abrogated by point mutation in the core binding sites of the EZH2 3'-UTR. A similar trend was also found in the SW620 cells (Fig. 4F). These results indicate that miR-494 exerts an inhibitory effect on PTEN expression via interaction with the 3'-UTR of PTEN in CRC cells.

\section{Discussion}

Several miRNAs have been identified as candidate components of oncogene and tumor suppressor networks in CRC, and these miRNAs and their targets play critical roles in carcinogenesis and are important for finding out novel therapeutic targets. Previous reports showed that miR-494 was downregulated and functioned as a tumor suppressor in human cholangiocarcinoma (21), 
gastric cancer (23) and lung cancer (28). However, other studies demonstrated that upregulation of miR-494 was associated with several types of human malignant solid tumors, including hepatocellular carcinoma (29), non-small cell lung cancer (30), and carcinoma induced by anti-BPDE (27). In these types of cancer, miR-494 seemed to be an oncogene, and promoted tumor cell proliferation, cell cycle, cell migration and invasion via regulation of the target genes PTEN, BIM and MCC. However, the function and clinical relevance of miR-494 in CRC have not yet been studied. In the current study, our results showed that miR-494 was upregulated in CRC tissues compared to adjacent tissues, and miR-494 was also upregulated in the tissues of CRC patients with lymph node metastasis compared with those without lymph node metastasis. Accordingly, we confirmed that miR-494 was commonly overexpressed in CRC cell lines compared with the normal colon epithelium cell line. These findings suggest that miR-494 may have vital roles in CRC progression.

We then investigated the clinical relevance of miR-494 in CRC. It was found that the increased expression of miR-494 in CRC was significantly associated with the adverse clinicopathological and histopathological features. Moreover, we demonstrated that the miR-494 expression level was also predictive of disease progression and cancer-specific death. Our results showed that the patients with high miR-494 level are usually at a significantly higher risk of cancer progression, cancer-specific death and shorter progression-free and overall survival time. Multivariate analysis demonstrated that high expression of miR-494 was a statistically significant risk factor affecting both progression-free and overall survival in CRC patients, which indicated that miR-494 expression could be an independent predictor of CRC progression and prognosis. The above results provided the first evidence supporting that miR-494 was a predictor of poor prognosis in CRC.

To reveal the role of miR-494 in CRC cells, we tested the effect of miR-494 on cell migration and invasion. Our results showed that miR-494 could promote cell migration and invasion. These results indicate that miR-494 might be a novel oncogenic miRNA that play important roles in the regulation of tumor metastasis of CRC. To address the molecular mechanisms involved in miR-494-mediated changes of biological properties, the PTEN was selected for further study because it was predicted to be a target of miR-494 by bioinformatics analysis. We found the following evidence supporting that miR-494 may be involved the modulation of PTEN expression. Both the mRNA and protein levels of PTEN were significantly downregulated after overexpression of miR-494 in SW480 cell lines. Conversely, the expression of PTEN was significantly upregulated after knockdown of miR-494 expression in SW620 cell lines. Furthermore, the mRNA levels of miR-494 inversely correlated with PTEN levels in CRC tissues. Moreover, overexpression of miR-494 decreased the luciferase reporter activity of WT 3'-UTR but not MT 3'-UTR of PTEN. These data confirm that PTEN is a downstream mediator of miR-494 function in CRC.

The PTEN gene, known also as mutated in multiple advanced cancer $1(M M A C 1)$, is a tumor suppressor gene located at chromosome 10q23.31 (31). The PTEN protein is principally involved in homeostatic maintenance of PI3K/Akt signaling originating from EGFR activation. PTEN/PI3K/Akt is highly involved in carcinogenesis and associated with EMT, cell cycle arrest (32-34). It has been also confirmed that PTEN, which counteracts PI3K/Akt activity, is involved in inhibition of cell proliferation and invasion (35). Thus, loss of PTEN function has an important impact on multiple aspects of cancer development such as cell proliferation, apoptosis resistance, angiogenesis, metabolism regulation, genomic instability, stem cell self-renewal, cell migration and invasion (36-39). It was reported that loss of PTEN expression was associated with liver metastasis, EMT, and enhanced migration and invasion of CRC $(25,26)$. Our results showed that miR-494 promoted tumor migration and invasion, and miR-494 upregulation was correlated with PTEN downregulation in CRC. These results indicate that upregulation of miR-94 in CRC may enhance cell migration and invasion, at least partially through the downregulation of PTEN expression.

The role of miR-494 in tumor development proves to be tissue dependent, causing increased proliferation in H460 lung cancer cells, hepatocellular carcinoma, breast and transformed bronchial epithelial cells $(19,27,29,30)$. However, it induces cell cycle arrest in lung cancer and cholangiocarcinoma $(22,28)$. Our results demonstrated that miR-494 increases cell migration and invasion in CRC, which indicated an oncogenic role of miR-494 in CRC. Moreover, our study supported the role of PTEN which was the direct target of oncogenic miR-494 as the tumor suppressor in CRC. In the tissues of CRC patients, we further confirmed the oncogenic role of miR-494 in CRC and its inverse relationship with PTEN. These results shed new light on the role of miR-494 in different types of tumors, especially in CRC.

Collectively, we showed that miR-494 was upregulated and its target PTEN was downregulated in CRC tissues. Moreover, upregulated miR-49 was significantly associated with adverse clinicopathological and histopathological features of CRC. We found ectopic expression of miR-494 could significantly promote cell migration and invasion in CRC cells through directly targeting PTEN. These results suggested that miR-494 played a role in inhibiting the development and progression of CRC by targeting PTEN and may potentially lead to a novel strategy for treatment of CRC.

\section{Acknowledgements}

The authors thank the local doctors and the patients who participated in our study.

\section{References}

1. Siegel R, Ma J, Zou Z and Jemal A: Cancer statistics, 2014. CA Cancer J Clin 64: 9-29, 2014.

2. van den Berg A, Mols J and Han J: RISC-target interaction: cleavage and translational suppression. Biochim Biophys Acta 1779: 668-677, 2008.

3. Calin GA and Croce CM: MicroRNA signatures in human cancers. Nat Rev Cancer 6: 857-866, 2006.

4. Kumar MS, Lu J, Mercer KL, Golub TR and Jacks T: Impaired microRNA processing enhances cellular transformation and tumorigenesis. Nat Genet 39: 673-677, 2007.

5. Bartel DP: MicroRNAs: genomics, biogenesis, mechanism, and function. Cell 116: 281-297, 2004.

6. Croce CM and Calin GA: miRNAs, cancer, and stem cell division. Cell 122: 6-7, 2005.

7. Gregory RI and Shiekhattar R: MicroRNA biogenesis and cancer. Cancer Res 65: 3509-3512, 2005. 
8. Lu J, Getz G, Miska EA, et al: MicroRNA expression profiles classify human cancers. Nature 435: 834-838, 2005.

9. Volinia S, Calin GA, Liu CG, et al: A microRNA expression signature of human solid tumors defines cancer gene targets. Proc Natl Acad Sci USA 103: 2257-2261, 2006.

10. Lujambio A, Calin GA, Villanueva A, et al: A microRNA DNA methylation signature for human cancer metastasis. Proc Natl Acad Sci USA 105: 13556-13561, 2008.

11. Ma Y, Zhang P, Wang F, et al: miR-150 as a potential biomarker associated with prognosis and therapeutic outcome in colorecta cancer. Gut 61: 1447-1453, 2012.

12. Almeida MI, Nicoloso MS, Zeng L, et al: Strand-specific miR-28-5p and miR-28-3p have distinct effects in colorectal cancer cells. Gastroenterology 142: 886-896, e9, 2012.

13. Pichler M, Ress AL, Winter E, et al: MiR-200a regulates epithelial to mesenchymal transition-related gene expression and determines prognosis in colorectal cancer patients. Br J Cancer 110: 1614-1621, 2014

14. Hwang WL, Jiang JK, Yang SH, et al: MicroRNA-146a directs the symmetric division of Snail-dominant colorectal cancer stem cells. Nat Cell Biol 16: 268-280, 2014.

15. Yang X, Zeng Z, Hou Y, et al: MicroRNA-92a as a potential biomarker in diagnosis of colorectal cancer: a systematic review and meta-analysis. PLoS One 9: e88745, 2014.

16. Liu Y, Zhou Y, Feng X, et al: Low expression of microRNA-126 is associated with poor prognosis in colorectal cancer. Genes Chromosomes Cancer 53: 358-365, 2014.

17. Zhao JJ, Yang J, Lin J, et al: Identification of miRNAs associated with tumorigenesis of retinoblastoma by miRNA microarray analysis. Childs Nerv Syst 25: 13-20, 2009

18. Shen YL, Jiang YG, Greenlee AR, Zhou LL and Liu LH: MicroRNA expression profiles and miR-10a target in anti-benzo[a] pyrene-7, 8-diol-9, 10-epoxide-transformed human 16HBE cells. Biomed Environ Sci 22: 14-21, 2009.

19. Liu Y, Lai L, Chen Q, et al: MicroRNA-494 is required for the accumulation and functions of tumor-expanded myeloid-derived suppressor cells via targeting of PTEN. J Immunol 188: 5500-5510, 2012.

20. Kwak SY, Yang JS, Kim BY, Bae IH and Han YH: Ionizing radiation-inducible miR-494 promotes glioma cell invasion through EGFR stabilization by targeting p190B rhoGAP. Biochim Biophys Acta 1843: 508-516, 2014.

21. Yamanaka S, Campbell NR, An F, et al: Coordinated effects of microRNA-494 induce $\mathrm{G}_{2} / \mathrm{M}$ arrest in human cholangiocarcinoma. Cell Cycle 11: 2729-2738, 2012.

22. Olaru AV, Ghiaur G, Yamanaka S, et al: MicroRNA downregulated in human cholangiocarcinoma control cell cycle through multiple targets involved in the G1/S checkpoint. Hepatology 54 2089-2098, 2011.

23. Li X, Luo F, Li Q, et al: Identification of new aberrantly expressed miRNAs in intestinal-type gastric cancer and its clinical significance. Oncol Rep 26: 1431-1439, 2011.
24. Leibovitz A, Stinson JC, McCombs WB 3rd, McCoy CE Mazur KC and Mabry ND: Classification of human colorectal adenocarcinoma cell lines. Cancer Res 36: 4562-4569, 1976.

25. Sawai H, Yasuda A, Ochi N, et al: Loss of PTEN expression is associated with colorectal cancer liver metastasis and poor patient survival. BMC Gastroenterol 8: 56, 2008.

26. Bowen KA, Doan HQ, Zhou BP, et al: PTEN loss induces epithelial-mesenchymal transition in human colon cancer cells. Anticancer Res 29: 4439-4449, 2009.

27. Liu L, Jiang Y, Zhang H, Greenlee AR and Han Z: Overexpressed miR-494 down-regulates PTEN gene expression in cells transformed by anti-benzo(a)pyrene-trans-7,8-dihydrodiol-9,10-epoxide. Life Sci 86: 192-198, 2010.

28. Ohdaira H, Sekiguchi M, Miyata K and Yoshida K: MicroRNA-494 suppresses cell proliferation and induces senescence in A549 lung cancer cells. Cell Prolif 45: 32-38, 2012

29. Lim L, Balakrishnan A, Huskey N, et al: MicroRNA-494 within an oncogenic microRNA megacluster regulates G1/S transition in liver tumorigenesis through suppression of mutated in colorectal cancer. Hepatology 59: 202-215, 2014

30. Romano G, Acunzo M, Garofalo M, et al: MiR-494 is regulated by ERK1/2 and modulates TRAIL-induced apoptosis in non-small-cell lung cancer through BIM down-regulation. Proc Natl Acad Sci USA 109: 16570-16575, 2012.

31. Molinari F and Frattini M: Functions and regulation of the PTEN gene in colorectal cancer. Front Oncol 3: 326, 2014.

32. Sansal I and Sellers WR: The biology and clinical relevance of the PTEN tumor suppressor pathway. J Clin Oncol 22: 2954-2963, 2004

33. Castellino RC and Durden DL: Mechanisms of disease: the PI3K-Akt-PTEN signaling node - an intercept point for the control of angiogenesis in brain tumors. Nat Clin Pract Neurol 3: 682-693, 2007.

34. Grille SJ, Bellacosa A, Upson J, et al: The protein kinase Akt induces epithelial mesenchymal transition and promotes enhanced motility and invasiveness of squamous cell carcinoma lines. Cancer Res 63: 2172-2178, 2003.

35. Vogt PK, Gymnopoulos M and Hart JR: PI 3-kinase and cancer: changing accents. Curr Opin Genet Dev 19: 12-17, 2009.

36. Zhang S and Yu D: PI(3)king apart PTEN's role in cancer. Clin Cancer Res 16: 4325-4330, 2010.

37. Song MS, Salmena L and Pandolfi PP: The functions and regulation of the PTEN tumour suppressor. Nat Rev Mol Cell Biol 13: 283-296, 2012.

38. Stewart AL, Mhashilkar AM, Yang XH, et al: PI3 kinase blockade by Ad-PTEN inhibits invasion and induces apoptosis in RGP and metastatic melanoma cells. Mol Med 8: 451-461, 2002.

39. Tamura M, Gu J, Matsumoto K, Aota S, Parsons R and Yamada KM: Inhibition of cell migration, spreading, and focal adhesions by tumor suppressor PTEN. Science 280: 1614-1617, 1998. 